\title{
Application of the finite difference method for modeling cantilever bar vibrations
}

\author{
M I Volnikov ${ }^{1, *}$ \\ ${ }^{1}$ Penza State Technological University, Baidukova Proezd/ ul. Gagarina, 1a/11, 440039, Penza, \\ Russia
}

\begin{abstract}
The paper is devoted to mathematical modeling of cantilever bars using the finite difference method. This method is widely used in structural mechanics for solving static problems. The novelty lies in the application of the finite difference method to simulate the dynamics of free and forced vibrations of the cantilever. Models have been developed that allow calculating the static and dynamic deflections of the cantilevers during free and forced vibrations, as well as simulating the vibrations of cantilever beams with attached vibration dampers. The resulting models of cantilever structures make it easy to modify system parameters, external influences and damping elements. All calculations were performed using the finite difference approach when moving along geometric and temporal coordinates.
\end{abstract}

\section{Introduction}

In applied and structural mechanics, for dynamic calculations of cantilever structures, a number of well-proven methods are used, both analytical ones based on the use of Krylov functions, and numerical ones, such as the grid method, the finite element method, the finite differences method [1-3]. The use of the analytical method based on the use of Krylov functions [4] makes it possible to study the dynamic processes of cantilevers with decent accuracy. However, the use of this method is accompanied by difficulties in the compilation of mathematical models and calculations when trying to modify the system or when introducing additional connections in it in the form of dynamic vibration dampers.

The use of numerical methods allows getting around this problem. The task that was posed when writing the paper was to find a simple and fairly effective method for modeling dynamic processes in cantilever bars. In our opinion, the most well-proven methods for these purposes are the finite element and finite difference methods (or the grid method). The finite element method is widely used for calculating complex objects under both static and dynamic loads. The finite difference method was used only for solving static problems in structural mechanics. However, in our opinion, the finite difference method is easier to use, therefore, an attempt was made in the work to apply the finite difference method to simulate the dynamics of free and forced oscillations of the cantilever. In addition, the advantages of this method include its versatility, which is much higher than that of analytical methods.

\footnotetext{
*Corresponding author: vmi1972@yandex.ru
} 


\section{Materials and methods}

The physical model of a cantilever bar with a dynamic vibration damper is shown in Figure 1. We will use the grid method to calculate the vibrations of the cantilever bar with a dynamic vibration damper (DVD). Let's replace the continuous physical model of the bar (Figure 1) with its discrete model in the form of a stepped cantilever and DVD. In this case, the bar is represented in the form of $n$ nodal points with lumped masses.

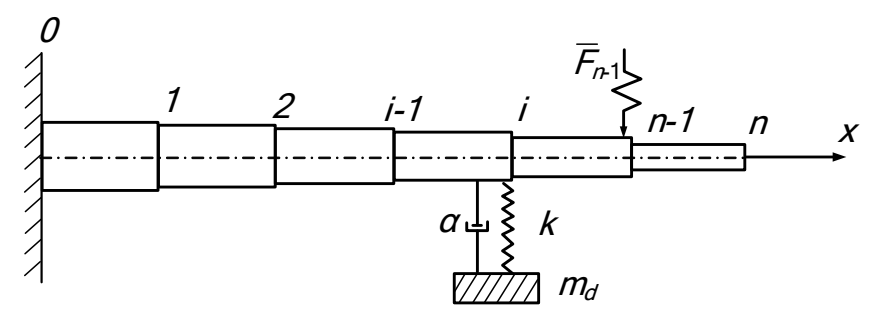

Fig. 1. Physical model.

We use a finite difference approach for movement along geometric and temporal coordinates [5]. The design and kinematic diagrams are shown in Figures 2-3, respectively [6,7].

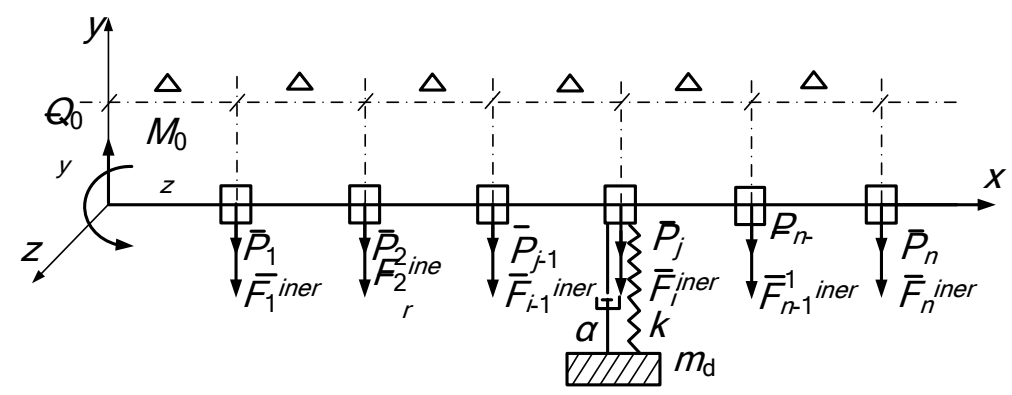

Fig. 2. Design diagram.

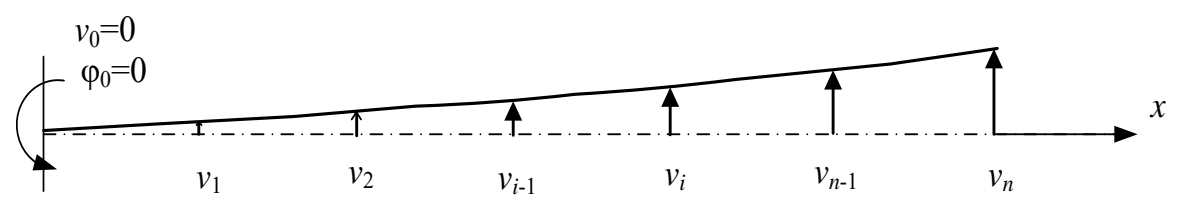

Fig. 3. Kinematic diagram.

\section{Static model}

The static equilibrium equations for a cantilever with nodes has the form [7]:

$$
\frac{E J_{i}}{\Delta^{2}} v_{i}^{\prime \prime}+M_{0 z}-i \Delta Q_{0 y}+\Delta \sum_{j=0}^{i-1} j P_{j}=0
$$

where $J$ - axial moment of inertia of sections, $E$ - elastic modulus, $M_{0 z}$ - moment in sealing, $Q_{0 y}$ - reaction force in sealing, $P_{j}$ - weight in $j$-section, $i$ takes values from $1, \ldots$ $n-1$, where $i$ is the number of the nodal point. 
In the central difference form, the second derivative of the deflections has the form

$$
v_{i}^{\prime \prime}=\frac{\left(v_{i-1}-2 v_{i}+v_{i+1}\right)}{\Delta^{2}}
$$

Let's divide the mass of the bar into 6 nodal points. (The modeling accuracy depends on the number of nodal points).

Boundary conditions for sealed end:

$$
\phi_{0}=v_{0}^{\prime}=\frac{1}{12 \Delta}\left(-25 v_{0}+48 v_{1}-36 v_{2}+16 v_{3}-3 v_{4}\right)=0
$$

where $\phi_{0}$ - bending angle in sealing.

To simplify the calculations, consider a bar with a constant cross section. In this case $P_{i}=P=9.8 \cdot m_{i}, l=6 \Delta$

The system of equations for $J_{i}=J=$ const has the form $\bar{a} \bar{x}=\bar{b}$, $A=\frac{E J}{\Delta^{2}}$.

$$
\bar{a}=\left(\begin{array}{cccccccc}
-2 A & A & 0 & 0 & 0 & 0 & 1 & -\Delta \\
A & -2 A & A & 0 & 0 & 0 & 1 & -2 \Delta \\
0 & A & -2 A & A & 0 & 0 & 1 & -3 \Delta \\
0 & 0 & A & -2 A & A & 0 & 1 & -4 \Delta \\
0 & 0 & 0 & A & -2 A & A & 1 & -5 \Delta \\
0 & 0 & 0 & 0 & 0 & 0 & 0 & 1 \\
0 & 0 & 0 & 0 & 0 & 0 & 1 & -6 \Delta \\
48 & -36 & 16 & -3 & 0 & 0 & 0 & 0
\end{array}\right), \bar{x}=\left(\begin{array}{c}
v_{1} \\
v_{2} \\
v_{3} \\
v_{4} \\
v_{5} \\
v_{6} \\
M_{o z} \\
Q_{0 y}
\end{array}\right), \bar{b}=\left(\begin{array}{c}
0 \\
-\Delta p \\
-3 \Delta p \\
-6 \Delta p \\
-10 \Delta p \\
6 p \\
-15 \Delta p \\
0
\end{array}\right) .
$$

From equations (4) it is easy to find the vector of values of static deflections $\bar{v}$.

As a practical application of the obtained models, hereinafter in the text, all calculations will be carried out for a bar of constant cross-section with the following parameters:

rod length $l=200 \mathrm{~mm}$; modulus of elasticity $E=2 \cdot 10^{11} \mathrm{~Pa}$; axial moment $J=8.2 \cdot 10^{-10} \mathrm{~m}^{4}$; bar weight $m_{\text {bar }}=77.5 \mathrm{~g}$; concentrated mass at node $m_{i}=77.5 / 6=12.92 \mathrm{~g}$.

\section{Modeling vibrations without a dynamic damper}

\subsection{Free vibration of the cantilever}

To simulate free vibrations of a bar, we add inertial forces to the static equilibrium equations $F_{i}^{i n e r}=-m_{i} \ddot{v}_{i}$ and their moments $M_{i}^{i n e r}=\sum_{i=1}^{5} \Delta(i-1) F_{i}^{i n e r}$.

We represent the forces of inertia in the central-difference form. The equation has the form 


$$
F_{i}^{i n e r}=\frac{m_{i}}{(\Delta t)^{2}}\left(v_{i, t-1}-2 v_{i, t}+v_{i, t+1}\right) .
$$

Then the equations of free vibrations of the cantilever take the form:

$$
\begin{gathered}
\frac{E J_{i}}{\Delta^{2}} v_{i}^{\prime \prime}+M_{0 z}-i \Delta Q_{0 y}+\Delta \sum_{j=0}^{i-1} j P_{j}+M_{i}^{i n e r}=0, \quad i=1, \ldots n-1 \\
Q_{0 y}-\sum_{j=1}^{n} P_{j}+\sum_{i=1}^{\mathrm{n}} F_{i}^{i n e r}=0 \\
M_{0 z}-l Q_{0 y}+\Delta \sum_{j=1}^{n-1}(n-j) P_{j}+\Delta \sum_{i=1}^{n-1}(n-i) F_{i}^{i n e r}=0 \\
\phi_{0}=v_{0}^{\prime}=\frac{1}{12 \Delta}\left(-25 v_{0}+48 v_{1}-36 v_{2}+16 v_{3}-3 v_{4}\right)=0
\end{gathered}
$$

Let's set the initial conditions of motion.

1) $v_{i, 0}=2 v_{i b a r}$, where $v_{b a r}$-displacement at $F_{i}=0$

$$
\text { 2) } \dot{v}_{i, 0}=0 \text { or } \frac{v_{i, 1}-v_{i, 0}}{\Delta t}=0 \Rightarrow v_{i, 1}=v_{i, 0}
$$

In matrix form, equations (5) taking into account (2) have the form:

$$
\bar{a} \bar{x}=\bar{b},
$$

where

$$
\bar{a}=\left(\begin{array}{cccccccc}
-2 A & A & 0 & 0 & 0 & 0 & 1 & -\Delta \\
A+\frac{m \Delta}{t^{2}} & -2 A & A & 0 & 0 & 0 & 1 & -2 \Delta \\
\frac{2 m \Delta}{t^{2}} & A+\frac{m \Delta}{t^{2}} & -2 A & A & 0 & 0 & 1 & -3 \Delta \\
\frac{3 m \Delta}{t^{2}} & \frac{2 m \Delta}{t^{2}} & A+\frac{m \Delta}{t^{2}} & -2 A & A & 0 & 1 & -4 \Delta \\
\frac{4 m \Delta}{t^{2}} & \frac{3 m \Delta}{t^{2}} & \frac{2 m \Delta}{t^{2}} & A+\frac{m \Delta}{t^{2}} & -2 A & A & 1 & -5 \Delta \\
-\frac{m}{t^{2}} & -\frac{m}{t^{2}} & -\frac{m}{t^{2}} & -\frac{m}{t^{2}} & -\frac{m}{t^{2}} & -\frac{m}{t^{2}} & 0 & 1 \\
\frac{5 m \Delta}{t^{2}} & \frac{4 m \Delta}{t^{2}} & \frac{3 m \Delta}{t^{2}} & \frac{2 m \Delta}{t^{2}} & \frac{m \Delta}{t^{2}} & 0 & 1 & -6 \Delta \\
-38 & -36 & -3 & 0 & 0 & 0 & 0
\end{array}\right),
$$




$$
\bar{b}=\left(\begin{array}{c}
0 \\
-\Delta P-\frac{m \Delta\left(v_{01}-2 v_{1}\right)}{t^{2}} \\
-3 \Delta P-\frac{2 m \Delta\left(v_{01}-2 v_{1}\right)}{t^{2}}-\frac{m \Delta\left(v_{02}-2 v_{2}\right)}{t^{2}} \\
-6 \Delta P-\frac{3 m \Delta\left(v_{01}-2 v_{1}\right)}{t^{2}}-\frac{2 m \Delta\left(v_{02}-2 v_{2}\right)}{t^{2}}-\frac{m \Delta\left(v_{03}-2 v_{3}\right)}{t^{2}} \\
-10 \Delta P-\frac{4 m \Delta\left(v_{01}-2 v_{1}\right)}{t^{2}}-\frac{3 m \Delta\left(v_{02}-2 v_{2}\right)}{t^{2}}-\frac{2 m \Delta\left(v_{03}-2 v_{3}\right)}{t^{2}}-\frac{m \Delta\left(v_{04}-2 v_{4}\right)}{t^{2}} \\
6 P+\frac{m}{t^{2}}\left(\left(v_{01}-v_{1}\right)+\left(v_{02}-v_{2}\right)+\left(v_{03}-v_{3}\right)+\left(v_{04}-v_{4}\right)+\left(v_{05}-v_{5}\right)+\left(v_{06}-v_{6}\right)\right) \\
-15 \Delta P-\frac{m \Delta\left(v_{05}-2 v_{5}\right)}{t^{2}}-\frac{2 m \Delta\left(v_{04}-2 v_{4}\right)}{t^{2}}-\frac{3 m \Delta\left(v_{03}-2 v_{3}\right)}{t^{2}}-\frac{4 m \Delta\left(v_{02}-2 v_{2}\right)}{t^{2}}-\frac{5 m \Delta\left(v_{01}-2 v_{1}\right)}{t^{2}}
\end{array}\right)
$$

Solving the system of equations (6), we find the vector of values of dynamic deflections $\bar{v}$.

\subsection{Modeling of forced vibrations}

Let's consider a model for describing forced vibrations of the cantilever [2]. In this case, a harmonically changing load will act on the end of the cantilever.

$$
F=F_{0} \cos \left(a \frac{2 \pi \tau}{T}\right)
$$

where $\frac{2 \pi}{T}=\omega-$ the circular frequency of the disturbing force, $a-$ a whole factor, $F_{0}=P$ - the amplitude of the disturbing force.

Let us add expressions for the driving force and its moments to the system of equations. We apply force to the end of the cantilever, then the moment is in the seal $M_{n}(F)=0$. The system of equations describing the dynamics of the oscillatory process will take the form:

$$
\begin{gathered}
\frac{E J_{i}}{\Delta^{2}} v_{i}^{\prime \prime}+M_{0 z}-i \Delta Q_{0 y}+\Delta \sum_{j=0}^{i-1} j P_{j}+M_{i}^{\text {iner }}=0, \quad i=1, \ldots n-1 \\
Q_{0 y}-\sum_{j=1}^{n} P_{j}+\sum_{\mathrm{i}=1}^{\mathrm{n}} F_{i}^{\mathrm{u}}+F_{0} \cos \left(a \frac{2 \pi \tau}{T}\right)=0 \\
M_{0 z}-l Q_{0 y}+\Delta \sum_{j=1}^{n-1}(n-j) P_{j}+\Delta \sum_{i=1}^{n-1}(n-i) F_{i}^{i n e r}=0 \\
\phi_{0}=v_{0}^{\prime}=\frac{1}{12 \Delta}\left(-25 v_{0}+48 v_{1}-36 v_{2}+16 v_{3}-3 v_{4}\right)=0
\end{gathered}
$$

Frequency response of the oscillations of the end of the cantilever is shown in Figure 4. Here, $g=\omega / \omega_{0}$ is the natural frequency of the system, $v-$ the deviation in arbitrary units. 


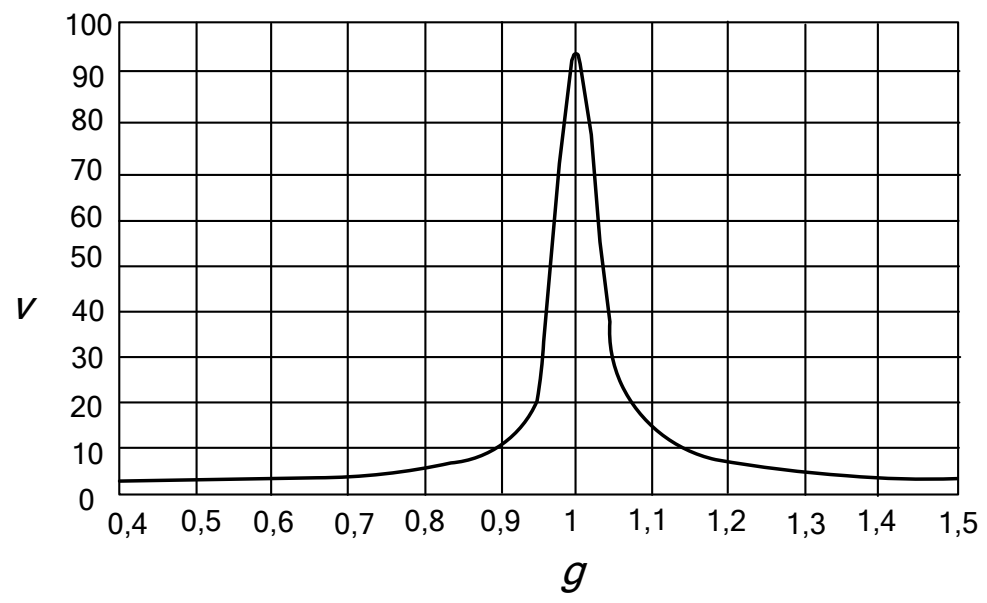

Fig. 4. Frequency response of the end of the cantilever in absolute terms.

\section{Modeling vibrations with a dynamic damper}

In most cases, bar structures are exposed to external influences. These can be wind or seismic loads or other disturbances. To suppress external disturbances affecting the cantilever bars, the technology uses dynamic vibration dampers. Let us simulate such structures using the finite difference method for the calculation.

\subsection{Application of a frictionless vibration damper}

Let's consider a simplified model of a dynamic vibration damper - without friction.

The equation describing the motion of the frictionless damper has the form [8]

$$
m_{7} \ddot{v}_{7}+k\left(v_{7}-v_{i}\right)=0 \text {, }
$$

where $v_{7}, v_{i}-$ deflections of the damper and deflection of the bar at the $i$-th point, $m_{7}-$ the mass of the damper.

In the central difference form, the equation of motion of the DVD will have the form

$$
m_{7}\left(\frac{v_{7, t-1}-2 v_{7, t}+v_{7, t+1}}{(\Delta t)^{2}}\right)+k\left(v_{7}-v_{i}\right)=0
$$

The damper is often tuned to the natural frequency of an oscillating system - a bar.

For modeling, we take the mass of the damper equal to 0.1 of the mass of the bar, i.e. $m_{7}=0.1 m_{\text {bar }}$.

The damper has a resonant frequency $\omega_{7}=\sqrt{k / m_{7}}$, therefore, the stiffness of the damper spring is:

$$
k=m_{7} \omega^{2}
$$

The system of equations in matrix form has the form $\bar{a} \bar{x}=\bar{b}$, where 


$$
\begin{aligned}
& \bar{a}=\left(\begin{array}{ccccccccc}
-2 A & A & 0 & 0 & 0 & 0 & 1 & -\Delta & 0 \\
A+\frac{m \Delta}{t^{2}} & -2 A & A & 0 & 0 & 0 & 1 & -2 \Delta & 0 \\
\frac{2 m \Delta}{t^{2}} & A+\frac{m \Delta}{t^{2}} & -2 A & A & 0 & 0 & 1 & -3 \Delta & 0 \\
\frac{3 m \Delta}{t^{2}} & \frac{2 m \Delta}{t^{2}} & A+\frac{m \Delta}{t^{2}} & -2 A & A & 0 & 1 & -4 \Delta & 0 \\
\frac{4 m \Delta}{t^{2}} & \frac{3 m \Delta}{t^{2}} & \frac{2 m \Delta}{t^{2}} & A+\frac{m \Delta}{t^{2}} & -2 A & A & 1 & -5 \Delta & 0 \\
-\frac{m}{t^{2}} & -\frac{m}{t^{2}} & -\frac{m}{t^{2}} & -\frac{m}{t^{2}} & -\frac{m}{t^{2}} & -\left(\frac{m}{t^{2}}+k\right) & 0 & 1 & k \\
\frac{5 m \Delta}{t^{2}} & \frac{4 m \Delta}{t^{2}} & \frac{3 m \Delta}{t^{2}} & \frac{2 m \Delta}{t^{2}} & \frac{m \Delta}{t^{2}} & 0 & 1 & -6 \Delta & 0 \\
48 & -36 & 16 & -3 & 0 & 0 & 0 & 0 & 0 \\
0 & 0 & 0 & 0 & 0 & -k & 0 & 0 & \frac{m_{7}}{t^{2}}+k
\end{array}\right) \\
& \bar{x}=\left\{v_{1}, v_{2}, v_{3}, v_{4}, v_{5}, v_{6}, M_{0}, Q_{0}, v_{7}\right\}^{T}, \\
& \bar{b}=\left(\begin{array}{c}
0 \\
-\Delta P-\frac{m \Delta\left(v_{01}-2 v_{1}\right)}{t^{2}} \\
-3 \Delta P-\frac{2 m \Delta\left(v_{01}-2 v_{1}\right)}{t^{2}}-\frac{m \Delta\left(v_{02}-2 v_{2}\right)}{t^{2}} \\
-6 \Delta P-\frac{3 m \Delta\left(v_{01}-2 v_{1}\right)}{t^{2}}-\frac{2 m \Delta\left(v_{02}-2 v_{2}\right)}{t^{2}}-\frac{m \Delta\left(v_{03}-2 v_{3}\right)}{t^{2}} \\
-10 \Delta P-\frac{4 m \Delta\left(v_{01}-2 v_{1}\right)}{t^{2}}-\frac{3 m \Delta\left(v_{02}-2 v_{2}\right)}{t^{2}}-\frac{2 m \Delta\left(v_{03}-2 v_{3}\right)}{t^{2}}-\frac{m \Delta\left(v_{04}-2 v_{4}\right)}{t^{2}} \\
6 P+\frac{m}{t^{2}}\left(\left(v_{01}-v_{1}\right)+\left(v_{02}-v_{2}\right)+\left(v_{03}-v_{3}\right)+\left(v_{04}-v_{4}\right)+\left(v_{05}-v_{5}\right)+\left(v_{06}-v_{6}\right)\right)+F_{0} \cos \left(a \frac{2 \pi \tau}{T}\right) \\
-15 \Delta P-\frac{m \Delta\left(v_{05}-2 v_{5}\right)}{t^{2}}-\frac{2 m \Delta\left(v_{04}-2 v_{4}\right)}{t^{2}}-\frac{3 m \Delta\left(v_{03}-2 v_{3}\right)}{t^{2}}-\frac{4 m \Delta\left(v_{02}-2 v_{2}\right)}{t^{2}}-\frac{5 m \Delta\left(v_{01}-2 v_{1}\right)}{t^{2}} \\
0 \\
-\frac{m_{7}}{t^{2}}\left(v_{07}-2 v_{7}\right)
\end{array}\right)
\end{aligned}
$$

Frequency response of the cantilever with a damper is shown in Figure 5.

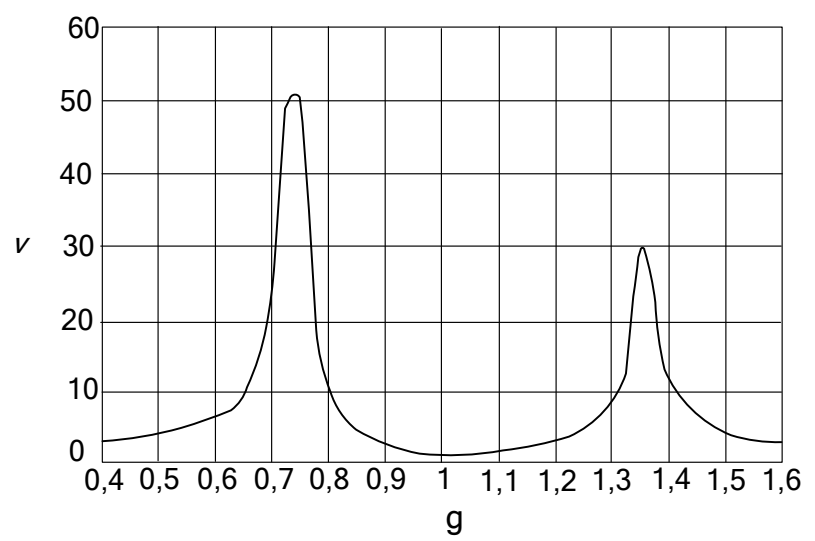

Fig. 5. Frequency response of the cantilever with a frictionless damper. 
As can be seen from the graphs, the vibration damper reduces the vibration amplitude in the resonance region $(\mathrm{g}=1)$, but this creates two resonance peaks on either side of the resonance frequency. The results obtained are in excellent agreement with the exact solutions obtained earlier in [9].

\subsection{Application of a vibration damper with friction}

In order to reduce the amplitudes of the peaks on both sides of the resonant frequency, a friction damper is used in practice. The dissipation of energy in the damper makes it possible to reduce the peak amplitudes. The vibration equation of the damper with friction has the form

$$
m_{7} \ddot{v}_{7}+k\left(v_{7}-v_{i}\right)+\alpha \dot{v}_{7}=0
$$

In central difference form

$$
m_{7}\left(\frac{v_{7, t-1}-2 v_{7, t}+v_{7, t+1}}{(\Delta t)^{2}}\right)+k\left(v_{7}-v_{i}\right)+\alpha\left(\frac{v_{7, t+1}-v_{7, t}}{\Delta t}\right)=0
$$

Resistance forces $F_{c}=\alpha \dot{v}$ will be added to the system of equations.

In matrix form, the system of equations has the form:

$$
\overline{a x}=\bar{b}
$$

where

$$
\bar{a}=\left(\begin{array}{ccccccccc}
-2 A & A & 0 & 0 & 0 & 0 & 1 & -\Delta & 0 \\
A+\frac{m \Delta}{t^{2}} & -2 A & A & 0 & 0 & 0 & 1 & -2 \Delta & 0 \\
\frac{2 m \Delta}{t^{2}} & A+\frac{m \Delta}{t^{2}} & -2 A & A & 0 & 0 & 1 & -3 \Delta & 0 \\
\frac{3 m \Delta}{t^{2}} & \frac{2 m \Delta}{t^{2}} & A+\frac{m \Delta}{t^{2}} & -2 A & A & 0 & 1 & -4 \Delta & 0 \\
\frac{4 m \Delta}{t^{2}} & \frac{3 m \Delta}{t^{2}} & \frac{2 m \Delta}{t^{2}} & A+\frac{m \Delta}{t^{2}} & -2 A & A & 1 & -5 \Delta & 0 \\
-\frac{m}{t^{2}} & -\frac{m}{t^{2}} & -\frac{m}{t^{2}} & -\frac{m}{t^{2}} & -\frac{m}{t^{2}} & -\left(\frac{m}{t^{2}}+k\right) & 0 & 1 & k+\frac{\alpha}{t} \\
\frac{5 m \Delta}{t^{2}} & \frac{4 m \Delta}{t^{2}} & \frac{3 m \Delta}{t^{2}} & \frac{2 m \Delta}{t^{2}} & \frac{m \Delta}{t^{2}} & 0 & 1 & -6 \Delta & 0 \\
0 & -36 & 16 & -3 & 0 & 0 & 0 & 0 & 0 \\
0 & 0 & 0 & 0 & 0 & -k & 0 & 0 & \frac{m_{7}}{t^{2}}+k+\frac{\alpha}{t}
\end{array}\right)
$$




$$
\bar{b}=\left(\begin{array}{c}
0 \\
-\Delta P-\frac{m \Delta\left(v_{01}-2 v_{1}\right)}{t^{2}} \\
-3 \Delta P-\frac{2 m \Delta\left(v_{01}-2 v_{1}\right)}{t^{2}}-\frac{m \Delta\left(v_{02}-2 v_{2}\right)}{t^{2}} \\
-6 \Delta P-\frac{3 m \Delta\left(v_{01}-2 v_{1}\right)}{t^{2}}-\frac{2 m \Delta\left(v_{02}-2 v_{2}\right)}{t^{2}}-\frac{m \Delta\left(v_{03}-2 v_{3}\right)}{t^{2}} \\
-10 \Delta P-\frac{4 m \Delta\left(v_{01}-2 v_{1}\right)}{t^{2}}-\frac{3 m \Delta\left(v_{02}-2 v_{2}\right)}{t^{2}}-\frac{2 m \Delta\left(v_{03}-2 v_{3}\right)}{t^{2}}-\frac{m \Delta\left(v_{04}-2 v_{4}\right)}{t^{2}} \\
6 P+\frac{m}{t^{2}}\left(\left(v_{01}-v_{1}\right)+\left(v_{02}-v_{2}\right)+\left(v_{03}-v_{3}\right)+\left(v_{04}-v_{4}\right)+\left(v_{05}-v_{5}\right)+\left(v_{06}-v_{6}\right)\right)+F_{0} \cos \left(a \frac{2 \pi \tau}{T}\right)+\frac{\alpha v_{7}}{\mathrm{t}} \\
-15 \Delta P-\frac{m \Delta\left(v_{05}-2 v_{5}\right)}{t^{2}}-\frac{2 m \Delta\left(v_{04}-2 v_{4}\right)}{t^{2}}-\frac{3 m \Delta\left(v_{03}-2 v_{3}\right)}{t^{2}}-\frac{4 m \Delta\left(v_{02}-2 v_{2}\right)}{t^{2}}-\frac{5 m \Delta\left(v_{01}-2 v_{1}\right)}{t^{2}} \\
0 \\
-\frac{m_{7}}{t^{2}}\left(v_{07}-2 v_{7}\right)+\frac{\alpha v_{7}}{\mathrm{t}}
\end{array}\right)
$$

Let us construct the frequency response of the cantilever vibrations with a friction damper with coefficients $\alpha=0.5$ and $\alpha=1.3$ (Figure 6).

It follows from the graphs that the vibration damper with friction is more effective. Internal friction in the damper increases energy dissipation and reduces resonance peaks. Thus, the operation of the oscillating system becomes more stable.

Figure 6 shows the frequency response of a cantilever structure without a vibration damper, as well as with attached frictionless and friction dampers, with coefficients $\alpha=0.5$ and $\alpha=1.3$.

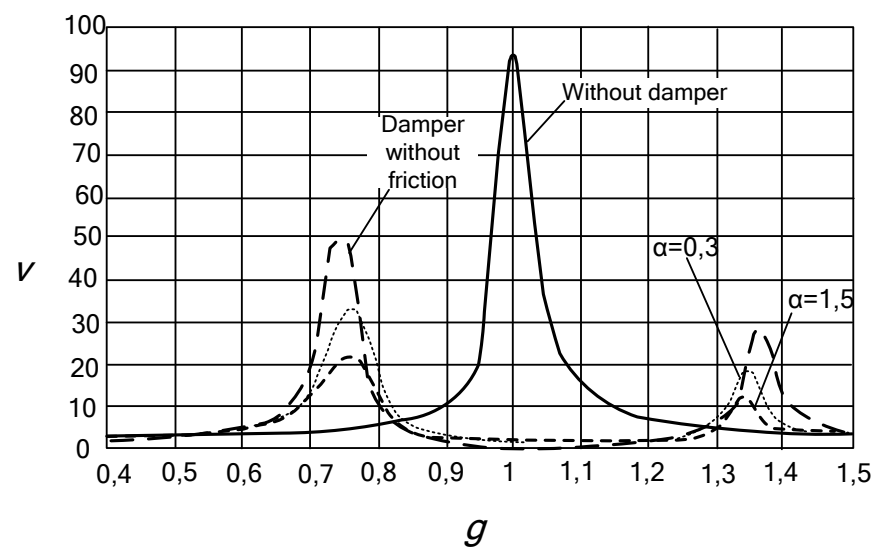

Fig. 6. Frequency response of the cantilever: without a damper, with a frictionless damper and with friction with different coefficients ( $\alpha=0.5$ and $\alpha=1.3$ ).

\section{Results and discussions}

The presented models allow studying:

1. Static deflections of cantilever bars (model (4));

2. The dynamics of the cantilever at free (6) and forced (8) vibrations. Determine the dynamic reactions of a cantilever bar to an external harmonic effect in the form of frequency response. 3. Dynamics of the cantilever with a vibration damper - models (11) and (14).

It will be interesting for the researcher to use the obtained results (14) for modeling the dynamics of a cantilever bar with different positions of the vibration damper. Figure 7 shows 
the dependences of the amplitude of vibrations of the end of the cantilever with different locations of the vibration damper along the length of the cantilever, made by the finite difference method.

As an example, Figure 7 shows the results of applying the obtained mathematical models (14). According to the results of the computational experiment, it was found that the damper installed at the end of the bar reduces the vibration amplitude by $2.2-4.3$ times, and the damper installed at the midpoint of the bar - by 3-4.8 times.

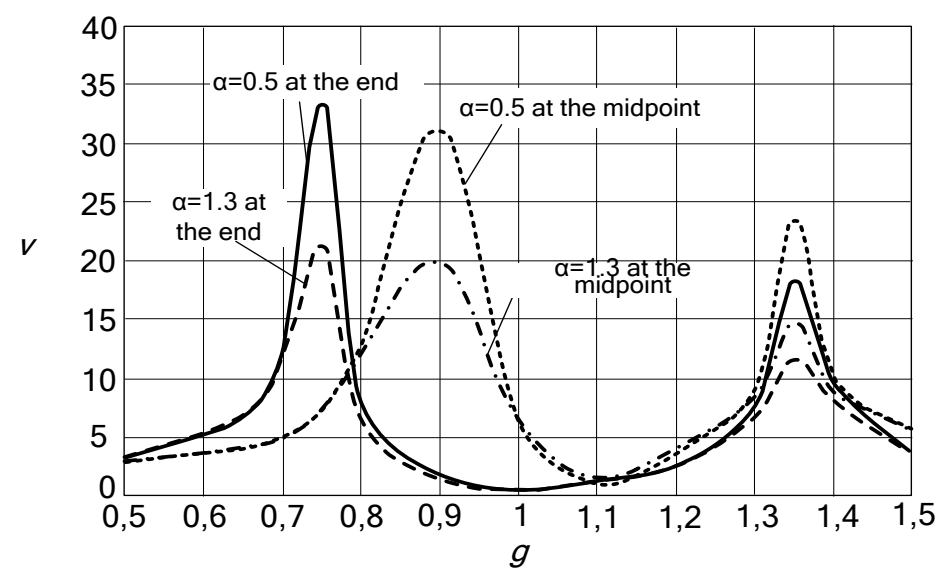

Fig. 7. Amplitudes of vibrations of the end of the cantilever with different positions of the vibration damper.

In further research, it is proposed to expand the use of the grid method for modeling dynamic processes in beam structures with multi-mass DVDs distributed along the entire length of the structure.

The application of the finite difference method is not limited to its application in cantilever structures. Changing the initial conditions in subsequent works, it is necessary to expand the area of application of the grid method to study the dynamics of not only bars with different end fixing, but also to other structures: cylindrical, plate, etc.

\section{Conclusions}

The use of the method of finite differences (grids) makes it easy to obtain the required model by adding additional parameters to the system, changing the conditions for the application of external disturbances and damping devices. These models are easy to upgrade, which allows studying a variety of dynamic processes in beam structures. Thus, model (14) becomes universal in application and can be used by researchers when modeling dynamic processes not only in cantilever beams of various sections, but also in other beam and plate structures.

\section{References}

1. Shein A I 2015 Modern scientific research and innovations. Part 13129

2. Shein A I 2002 Industrial and civil construction 227

3. Malik, A V 2018 Tomsk State University Journal. Mathematics and Mechanics 5488

4. Vinogradov Yu, Cauchy-Krylov I 2013 News of higher educational institutions. Mechanical engineering 815 
5. Volnikov M I 2007 Actual problems of modern construction: Proceedings of the international scientific and technical conference (Penza: PGUAS) p 95

6. Volnikov M I 2016 Modern problems of engineering and technology development: Proceedings of the international scientific and practical conference (Penza: PenzGTU) $p$ 127

7. Volnikov M I 2016 XXI century: Results of the past and problems of the present plus 5(33) 63

8. Volnikov M I 2016 Modern problems of engineering and technology development: Proceedings of the international scientific and practical conference (Penza: PenzGTU) $p$ 122

9. Ananiev I V 1965 Oscillations of elastic systems in aircraft structures and their damping (M.: Mechanical Engineering) p 526 